\title{
Goal tracking in attentional-associative networks: Spatial learning and the hippocampus
}

\author{
JOHN W. MOORE and KENNETH J. STICKNEY \\ University of Massachusetts, Amherst, Massachusetts
}

\begin{abstract}
Moore and Stickney's (1980) formalization of Mackintosh's (1975) attention theory is applied to the general problem of goal tracking in spatial learning tasks. The model is stated, and computer simulations describe the evolution of attentional-associative networks for various scenarios of goal tracking. Both normal and abnormal cases are considered, the latter involving the failure of computations that normally reduce the salience of stimuli and other events. The resulting behavioral pathology, when observed, is likened to animals with deficits in spatial learning as described by O'Keefe and Nadel (1978) in their theory of the hippocampus as a cognitive map.
\end{abstract}

This article describes how the authors' theory of attentional-associative networks (e.g., Moore \& Stickney, 1980) might be applied to goal-directed behavior (goal tracking). The specific application is to a problem of long-standing concern to psychologists, namely, that of understanding precisely what is learned within a goal-tracking task in which the goal is obscure and can be approached only through the use of cues or signs. Such an understanding is essential for predicting the nature of behavioral pathologies resulting from, for example, damage to the hippocampal formation. Although our model grew out of work in the area of traditional Pavlovian conditioning in animals, its extension to goal tracking suggests that it may ultimately prove applicable to other forms of "searching," for example, the problem of cued retrieval of information from an associative memory system.

\section{Background}

Moore and Stickney (1980) suggested that behavioral pathologies in learning and memory might be analyzed in terms of alterations in computations that go into the evolution of attentional-associative networks. Specifically, we suggested that organisms suffering ablations of the hippocampal formation cannot reduce the salience of cues that are poorly correlated with a reinforcer. For example, when an animal is confronted with a learning task that can be solved by either cue or place strategies (cf. Black, Nadel, \& O'Keefe, 1977), hippocampal ablations can lead to conflicting response tendencies because irrelevant cues compete with relevant cues in leading the

Preparation of this report was facilitated by NSF Grant BNS 8100322. The authors' complete address is: Department of Psychology, University of Massachusetts, Amherst, Massachusetts 01003. animal toward solution. The present paper spells out more precisely how this might happen within the system of equations and computations described in our previous paper. These equations and computations have undergone some modifications in the interim, the principal modification involving the incorporation into the theory of a mechanism for learned inhibition. The incorporation of rules of learned inhibition is primarily designed to account for phenomena of conditioned inhibition as observed in classical conditioning (Rescorla, 1979; Moore \& Stickney, Note 1).

From a longer perspective, the present article is an advance on points developed by Moore (1979). That paper treated at some length the way principles of modern associative learning theory might be applied as interpretative alternatives to portrayals of hippocampal functioning cast in terms of cognitive maps (Black et al., 1977; Moore \& Solomon, 1980; O’Keefe \& Nadel, 1978).

\section{Modern Theories of Learning}

One of the more exciting, yet unpublicized, developments in theoretical psychology over the past decade has been the emergence of a new generation of mathematical models of associative learning. Unlike the stochastic models of Bush and Mosteller (1955) and Estes (1959), and many others of that period, the new models are designed to account for phenomena of classical Pavlovian conditioning. Prominent examples of such models include Frey and Sear's (1978) cusp-catastrophe theory, Mackintosh's (1975) attention theory, and the Rescorla-Wagner (1972) model. Like the stochastic models of the earlier era these models appear to have potential for development into areas of human perception, learning, memory, and performance. This potential is particularly evident in some of the more recent models 
based on animal conditioning (e.g., Pearce \& Hall, 1980; Sutton \& Barto, 1981; Wagner, Note 2).

The trend in contemporary learning theory is away from S-R behaviorism and toward cognitive psychology. While cognitive psychology is concerned with the acquisition and structure of knowledge as related to, for example, perception and language, animal learning psychology is increasingly concerned with the acquisition and structure of knowledge as related to the performance of conditioned behavior. Such performance provides insights into the nature of knowledge acquired in a particular context, how it is acquired, stored, and "fed forward" to influence behavior. These concerns represent a throwback to the era of Tolman and others, who spoke of animals' possessing knowledge of relationships, cognitive maps, hypotheses, strategies, and other such mental paraphernalia.

It is not so much a question of whether the cognitive orientation is more valid than S-R behaviorism; it is more a question of how contemporary investigators in the field of animal learning can best influence the mainstream of psychological thought at its confluence with artificial intelligence and other computational sciences. Few would deny the contributions of traditional reinforcement theory or the continuing value of concern for real behavior in the presence of real stimuli and under specified antecedant conditions. At the same time, few would deny the potential rewards of attacking ageold questions of epistemology using animal systems - systems that may be brought into intimate contact with biology. Encouraging as these developments in animal learning theory are, there remains the problem of breaking away from "simple" associative learning tasks such as classical conditioning and applying current models to more complicated tasks such as those involving goal-directed behavior.

\section{Goal-Directed Behavior}

The problem of goal-directed behavior in the free field and in complex mazes gave rise to the cognitive theories of Tolman and his school. Much of the language of that period remains in contemporary usage. This is perhaps most evident in O'Keefe and Nadel's (1978) heroic synthesis of Tolman's theory of the cognitive map with the functions of the hippocampal formation of the brain. These authors cast the learning and performance of goal-directed behavior in terms of two potentially conflicting strategies-a spatial strategy, based on a Tolmanian cognitive map, and a cue strategy, based on principles of sign tracking. Impaired learning and performance of goal-directed behavior under hippocampal dysfunction is presumably due to the loss or unavailability of spatial strategies.

Space precludes delving too deeply into O'Keefe and Nadel's theory. Our purpose in citing it is to call attention to the gulf of theoretical sophistication that has separated the mathematically elegant and deterministic models based on classical conditioning from the purely verbal (and vague) conceptions of, for example, the cognitive map. If rigorous deterministic models of goal-directed behavior had been available, it is possible that O'Keefe and Nadel would have incorporated them into their theory of the hippocampus.

\section{Moore-Stickney (1980) Model}

Moore and Stickney's (1980) model of attentionalassociative networks is derived from Mackintosh's (1975) attention theory. Like Mackintosh's theory, our model addresses a variety of phenomena of classical conditioning, including latent inhibition and blocking.

One key idea of the model is that predictive associative relationships control behavior. For example, the associative relationship whereby a conditioned stimulus or cue predicts a reinforcer controls conditioned responding. Conditioned responses may be discrete reflexes or movement toward or away from the reinforcer.

A second key idea is that the rate of growth or decline of associative bonds depends primarily on the associability, or salience, of the conditioned stimulus or cue. For a given conditioned stimulus, salience may increase, decrease, or remain unchanged with experience. It will increase whenever (and to the extent that) it is the best available predictor of the reinforcer. In cases in which the predictive associative strength of the conditioned stimulus is equal to that of the best alternative, salience remains unchanged.

A third key idea is that the predictive associative relationship increases whenever the conditioned stimulus correctly predicts the reinforcer, and it decreases whenever it does not. The magnitude of these changes of associative strength depends on timing: If the reinforcer follows the conditioned stimulus at an interval of time optimal for association, the increase is greater than if the two events are separated by a longer interval or if they occur concurrently. Similarly, if the reinforcer does not occur following the conditioned response, the decrease in associative strength also depends on timing. The precise role of timing in increasing and decreasing associative strength is described more fully below.

A fourth key element of the model is that the salience of a conditioned stimulus, or of any stimulus for that matter, depends not only on predictive associative relationships with the nominal reinforcer, but on other predictive associative relationships as well. Thus, for example, the extent to which a cunditioned stimulus predicts other events in comparison with the best competing predictor present at the same time contributes to the computation of salience. This idea is developed fully in our previously published description of the model. For purposes of describing how the model might be applied to goal-directed be- 
havior, we assume for ease of exposition that it is only the prediction of the reinforcer (goal) that governs the salience of potential cues. This simplifying assumption does no violence to the ideas we wish to convey.

The final feature of the model worth noting is that it applies to "real time." That is, the computations involving predictive associative relationships and salience can occur continuously and not merely on arbitrarily designated trials. For our computer simulations, however, time is broken down into successive bins of equal duration.

\section{Antiassociations}

One limitation of our initial model (Moore \& Stickney, 1980) is its failure to incorporate rules for generating conditioned inhibition. For present purposes, conditioned inhibition refers to another kind of predictive association, namely, the association whereby a conditioned stimulus or cue predicts that the reinforcer will not occur. We designate this predictive relationship an association.

One key feature of the antiassociation is that it combines with the association to yield a net predictive association. The rule is simple-if a is the predictive association value with respect to the reinforcer and $b$ is the antiassociation, the net associative value is $a-b$.

A second key idea is that the antiassociation develops only in the presence of strong expectation or anticipation of the reinforcer. This means that if the reinforcer fails to follow the occurrence of a potential predictor, an antiassociation will begin to develop only if the sum of all the potential predictors of reinforcer present at that moment exceeds some threshold value.

The antiassociation allows the Moore-Stickney (1980) model to generate most of the behavioral phenomena of Pavlovian conditioned inhibition, thereby surmounting one limitation of our original model. We need not dwell on these phenomena, since to do so would be tangential to our present purpose.

\section{Formal Statement of the Model}

The equations governing the changes in predictive associations whereby the $i^{\text {th }}$ of a countable set of $R$ stimuli predicts the goal or reinforcer are as follows:

$$
\Delta \mathrm{V}_{\mathrm{i}}=\alpha_{\mathrm{i}} \theta \tau\left(1-\mathrm{V}_{\mathrm{i}}\right)
$$

whenever the goal follows the ith stimulus, and

$$
\Delta \mathrm{V}_{\mathrm{i}}=\alpha_{\mathrm{i}} \theta^{\prime} \tau\left(0-\mathrm{V}_{\mathrm{i}}\right)
$$

whenever the goal does not follow the ith stimulus.

The variable $V_{i}$ is the theoretical dependent variable representing associative strength or "value"; it is bound between 0 and 1 .
The parameter $\alpha_{\mathrm{i}}$ is the salience or associability of the ith stimulus for predicting the goal; it is bound between 0 and 1 .

The parameter $\theta(0<\theta<1)$ is the rate parameter contributed by the goal; it remains constant. The parameter $\theta^{\prime}\left(0<\theta^{\prime}<\theta\right)$ is the rate parameter contributed by the nonoccurrence of the goal.

Finally, $\tau$ is a function defined as follows:

$$
\tau=\tau(\Delta t)=\mathrm{e}^{\mathrm{k}(\mathrm{q}-\Delta \mathrm{t})}
$$

where $\Delta t \geqslant 0$. The constant $q$ is positive and equal to the interval optimal for association; $k(0<k<1)$ is a constant. The independent variable in Equation 3 is $\Delta t$, the time between the occurrence of the ith stimulus and the occurrence (or nonoccurrence) of the goal.

In our computer simulations, values of $\Delta t$ are expressed in units of size q. Thus, $\tau$ equals 1 when the ith stimulus and the goal are separated by $\Delta t=1 q$. When $\Delta t=0, \tau$ is assigned an arbitrarily small positive value, and when $\Delta t<0, \tau$ is set equal to 0 .

The equations for the antiassociation whereby the ith stimulus predicts that the goal will not occur are as follows:

$$
\Delta \overline{\mathbf{V}}_{\mathrm{i}}=\alpha_{\mathrm{i}} \theta^{\prime} \tau\left(1-\overline{\mathrm{V}}_{\mathrm{i}}\right)
$$

whenever the goal does not follow the ith stimulus and the sum of associative values of all stimuli present (countable set $\mathbf{S} \subset \mathbf{R}$, with index $\mathrm{j}$ including $\mathrm{i}$ ) exceeds some arbitrary and constant threshold $L$. Thus, the antiassociation of the ith stimulus $\left(\bar{V}_{\mathfrak{i}}\right)$ increases by Equation 4 whenever

$$
\mathbf{L}<\sum_{\mathbf{j \varepsilon S}} \mathbf{V}_{\mathbf{j}}
$$

The antiassociation decreases whenever the goal follows the ith stimulus according to Equation 5:

$$
\Delta \bar{V}_{i}=\alpha_{i} \theta \tau(0-\bar{V})
$$

The rate parameters of Equations 4 and 5 are the same as in Equations 1 and 2 , and $\tau$ is computed in precisely the same way as described in Equation 3.

All parameters are constant and independent of moment-to-moment changes of $V_{i}$ and $\bar{V}_{i}$ except for $\alpha_{i}$. This parameter increases according to the following equation when $V_{i}>V_{k}$ :

$$
\Delta \alpha_{i}=c\left(1-\alpha_{i}\right)\left\{V_{i}-V_{k}\right\},
$$

where $i, k \in S$, but $i \neq k$. If $V_{i} \leqslant V_{k}, \alpha_{i}$ decreases (or remains unchanged) according to Equation 7:

$$
\Delta \alpha_{i}=-\alpha_{i} c\left\{V_{k}-V_{i}\right\}
$$


The rate parameter $\mathrm{c}$ is a constant $(0<\mathrm{c}<1)$ and $\mathrm{V}_{\mathrm{k}}$ in Equations 6 and 7 is always the maximum of the set of associative values mapped onto $S$ excluding $V_{i}$. Thus, $V_{k}=\max V_{j}: j \varepsilon S, j \neq i$.

We conclude presentation of the formal model by defining the net associative value of the $i^{\text {th }}$ stimulus:

$$
\dot{\mathrm{V}}_{\mathrm{i}}=\mathrm{V}_{\mathrm{i}}-\overline{\mathrm{V}}_{\mathrm{i}}
$$

For some theoretical purposes, $\dot{\mathrm{V}}_{\mathrm{i}}$ and $\dot{\mathrm{V}}_{\mathrm{k}}$ may be substituted for $V_{i}$ and $V_{k}$ in Equations 6 and 7 . In other applications, the threshold $\mathrm{L}$ employed in connection with the growth of the antiassociation in Equation 5 might be constrained as follows:

$$
\mathbf{L}<\sum_{\text {jeS }} \dot{\mathbf{V}}
$$

\section{Goal-Tracking Among Places, Cues and Responses}

The introductory portion of this paper cited O'Keefe and Nadel's (1978) theory of the hippocampus as a cognitive map because it is within that frame of reference that we wish to apply Equations 1-8. We hope to show how, in the course of goal tracking in the free field, an animal such as laboratory rat evolves a relevant attentional-associative network. The mechanics of the model can best be illustrated by keeping things as simple as possible while at the same time integrating several important points.

The first point is that goal tracking occurs within a context or space featuring distinct fixed landmarks. Following O'Keefe and Nadel (1978; see also Black et al., 1977), these landmarks enable the animal to construct a representational system for the various places within this context. We further assume that the occurrence or nonoccurrence of the goal is a local event, that it is only when the animal is in the immediate locale of a particular place that the computations of Equations 1-8 occur. This will become clear as we proceed.

The second point is that the context contains various salient features or signs that are not fixed. These are potential clues to the occurrence or location of the goal. As with places, these signs (cues) enter into the computations only when they are proximal to the organism. After a "learning experience," both places and cues might enter into computations "from some distance."

For now, it is essential to appreciate the idea that a given cue or signal may be dissociated from a particular place. An example of what we mean would be a scarecrow in a field of new corn that the farmer moves periodically from place to place. The scarecrow (or man) is not confined to a particular place in the field or in the memory system of an experienced scavanging crow.

The final point is that instrumental conditioning plays an important role in goal tracking. That is, the occurrence and nonoccurrence of the goal is associated with (i.e., contingent upon) certain behaviors: locomoting or standing still, for example. This instrumental component can give rise to associationistically derived "beliefs" that locomoting (but in no particular direction) or standing still (but not in any particular place) are signals for the goal. An essential point to appreciate is that the animal can at any moment in time hold these two beliefs simultaneously. An animal that believes that locomoting will produce the goal and that standing still will also produce the goal is clearly in a conflict situation. This conflict, derived from mutually interfering associations, can only be resolved by the normal evolution of the attentional-associative network.

Another source of such conflict would arise when the animal, based on past experience, simultaneously "believes" that the goal is to be found near a cue located at one place while also "believing" that the goal is located at some other place. In short, we are dealing with a sort of shell game. As we shall see, certain neuropsychological pathologies, when tied to certain parameters and computations of the model, render resolution of such conflict difficult or impossible. This, too, will be illustrated below.

In the normal course of events, the conflicting "beliefs" confronting the goal tracker are resolved by balancing associations with antiassociations and then evolving a course of action (direction of movement) based on the net associative value (ن) with greatest value. Developing these ideas further is best accomplished by a few simple scenarios.

\section{Scenarios of Goal-Tracking Among Places, Cues, and Responses}

Three scenarios are considered, each consisting of two stages of training. Within each stage there are four repetitive epochs. Each epoch, in turn, is broken down into four bins of event patterns.

The events of each scenario are coded to ease the burden of too many subscripts. Here is the code corresponding to the eight events needed to make our point-the dramatis personae: $X=$ context; $A=a$ place (i.e., neighborhood of a point within the context); $B=$ another place; $C=a$ cue; $D=$ all other cues; $\mathrm{E}=$ response (e.g., locomotion); $\mathrm{F}=$ all responses incompatible with $\mathrm{E}$ (e.g., standing still); $\mathrm{G}=$ goal (assumed to be obscure and revealed only proximally). The computations of Equations 1-8 apply to each of these events so long as the animal is within the cuntext. We shall assume that computations are frozen as soon as the animal leaves, or is removed from, the context at the end of each epoch.

Episodes in which the animal enters and leaves the context are computation epochs, and each epoch consists of a succession of time bins of duration $q$ of Equation 3. Table 1 gives the sequence of 
Table 1

Sequence of Events in Scenario Consisting of Two Stages of Training, Each With Four Epochs of Four Bins

\begin{tabular}{|c|c|c|c|c|c|c|c|c|}
\hline \multirow[b]{2}{*}{ Event } & \multicolumn{4}{|c|}{$\begin{array}{c}\text { Bin of Each Epoch } \\
\text { of Stage } 1\end{array}$} & \multicolumn{4}{|c|}{$\begin{array}{c}\text { Bin of Each Epoch } \\
\text { of Stage } 2\end{array}$} \\
\hline & 1 & 2 & 3 & 4 & 1 & 2 & 3 & 4 \\
\hline X & $*$ & $*$ & $*$ & $*$ & $*$ & $*$ & $*$ & $*$ \\
\hline A & - & - & - & - & - & - & $*$ & $*$ \\
\hline B & - & - & $*$ & * & $*$ & - & - & - \\
\hline $\mathrm{C}$ & - & - & $*$ & * & - & - & $*$ & $*$ \\
\hline D & - & - & - & - & - & - & - & - \\
\hline $\mathrm{E}$ & - & $*$ & $*$ & - & - & $*$ & $*$ & - \\
\hline$F$ & $*$ & - & - & $*$ & $*$ & - & - & $*$ \\
\hline G & - & - & - & $*$ & - & - & - & $*$ \\
\hline
\end{tabular}

Note-An asterisk (*) denotes the presence of an event in a bin; a dash (-) denotes the absence of an event in a bin. The pattern and sequence of events in the table are those of ScenarioSequence 1. Scenario-Sequence 2 is the same except that Event B was excluded from Bin 1 of Stage 2 epochs. Scenario-Sequence 3 is the same except that Event $A$ was included in Bin 2 of Stage 1 epochs.

events as they actually unfold within epochs of the scenario. Computations across each bin of these epochs produce changes in the following variables: $\alpha, \mathrm{V}, \overline{\mathrm{V}}$, and $\dot{V}$ for each of the eight elements of the task.

The starting values of the eight $\alpha$ s is of vital importance in how the attentional-associative network unfolds over the epochs of our scenario. We initialized these eight $\alpha \mathrm{s}$ to be equal to .50 . All Vs, $\overline{\mathrm{V}} \mathrm{s}$, and $\dot{\mathrm{V}} \mathrm{s}$ were assumed to be equal to 0 initially. The other parameters, $c, \theta, \theta^{\prime}$, and $\tau$, were held constant and were equal for each of the eight elements of the task (see Table 2). Apart from Equation $3(\tau)$, there are a total of $7 \times 8=56$ computations possible at one time or another, that is, Equations 1-8, less Equation 3, for each element.

For the sake of brevity, we shall suppress some of the finer points of our computer simulations (see Moore \& Stickney, 1980, Note 1) and move ahead to a consideration of the fate of the variables of interest. These are given in Table 2, which gives the ter- minal values of $\dot{V}$ and $\alpha$ for each of the elements of the situation.

\section{Scenario Sequences}

The situation we wish to portray is one in which our goal-tracking animal first learns that the goal (G) occurs contiguously and in the locale or neighborhood of cue $C$, located at place $B$. The animal next learns that $G$ may be found near $C$, but at a different place, place A. The initial training experience occurs in Stage 1, and, as indicated, it consists of four identical epochs. Each epoch, in turn, consists of four time bins. The distribution of events into bins of width q (Equation 3) is shown in the left-hand portion of Table 1. The second training experience (Stage 2) is summarized in the right-hand portion of Table 1. It, too, consists of four identical epochs of four bins each.

The minor players have routine and uninteresting roles: $X$ (context) inevitably occurs in every bin of every epoch. "Other cues" (D) is not involved at all: the scenario requires only one cue, and that is $\mathrm{C}$. The animal's behavior, when considered independently of its direction, is monotonously the same in each epochbeginning with "standing still," followed for two bins by "locomoting," and closing with standing still once again (in consummation with the goal) as each epoch ends. The role of $\mathrm{C}$ is also monotonous, but significant to the plot: each epoch closes with the animal in the neighborhood of C (Bins 3-4).

The dramatic tension is between the two places, A and B. In Scenario-Sequence 1, A is not encountered until Stage 2 of training when $C$ is moved to this location. Place $B$ is encountered during each epoch of Stage 1 because this is where $C$ and the goal are located. In Stage 2, however, the animal spends the greatest portion of Bin 1 of each epoch near place B, presumably because this is where the goal had been located in Stage 1.

The animal enters the first stage of training with no associative links to the goal: all Vs are zero. In

Table 2

Net Values and Saliences $(\times 100)$ of Events at the End of Scenarios of Table 1

\begin{tabular}{|c|c|c|c|c|c|c|c|c|c|c|c|c|}
\hline \multirow[b]{3}{*}{ Event } & \multicolumn{4}{|c|}{ Scenario-Sequence 1} & \multicolumn{4}{|c|}{ Scenario-Sequence 2} & \multicolumn{4}{|c|}{ Scenario-Sequence 3} \\
\hline & \multicolumn{2}{|c|}{ Normal } & \multicolumn{2}{|c|}{ Hippocampal } & \multicolumn{2}{|c|}{ Normal } & \multicolumn{2}{|c|}{ Hippocampal } & \multicolumn{2}{|c|}{ Normal } & \multicolumn{2}{|c|}{ Hippocampa } \\
\hline & $\dot{\mathrm{V}}$ & $\alpha$ & $\dot{\mathrm{V}}$ & $\alpha$ & $\dot{\mathrm{V}}$ & $\alpha$ & $\dot{\mathrm{V}}$ & $\alpha$ & $\dot{\mathrm{V}}$ & $\alpha$ & $\dot{V}$ & $\alpha$ \\
\hline $\mathrm{X}$ & 48 & 33 & 49 & 50 & 47 & 18 & 49 & 50 & 45 & 16 & 49 & 50 \\
\hline A & 29 & 5 & 47 & 50 & 29 & 5 & 47 & 50 & 50 & 18 & 59 & 50 \\
\hline B & 36 & 50 & 35 & 50 & 47 & 50 & 47 & 50 & 36 & 50 & 36 & 50 \\
\hline $\mathrm{C}$ & 74 & 65 & 73 & 63 & 76 & 80 & 76 & 80 & 76 & 80 & 76 & 76 \\
\hline D & 0 & 50 & 0 & 50 & 0 & 50 & 0 & 50 & 0 & 50 & 0 & 50 \\
\hline $\mathbf{E}$ & 59 & 50 & 59 & 50 & 59 & 50 & 59 & 50 & 59 & 50 & 59 & 50 \\
\hline $\mathbf{F}$ & 9 & 1 & 7 & 50 & 9 & 0 & 7 & 50 & 8 & 0 & 7 & 50 \\
\hline $\mathbf{G}$ & 0 & 0 & 0 & 50 & 0 & 0 & 0 & 50 & 0 & 0 & 0 & 50 \\
\hline
\end{tabular}

Note-Values of constants: $k=.67, \tau(\Delta t=0)=.067, c=1, \theta=.30, \theta^{\prime}=.05$, and $L=.50$. Variables were initialized as follows: $\alpha_{X}=$ $\alpha_{A}=\alpha_{B}=\alpha_{C}=\alpha_{D}=\alpha_{E}=\alpha_{F}=\alpha_{G}=.50$, and $V_{X}=V_{A}=V_{B}=V_{C}=V_{D}=V_{E}=V_{F}=V_{G}=0 ;$ similarly, all $\bar{V} s$ and $\dot{V}$ s were zero initially. 
addition, all events-stimuli, places, responses-are equally associable with the goal; that is, all $\alpha$ s are (arbitrarily) equal to .50. On entering the situation for the first time, the animal "explores" the training environment until locating the goal near $C$ at place B. With each succeeding epoch, the predictive associative value of $C$ and $B$ increases, as does the value of the responses of locomoting $(E)$ and, to a much lesser extent, the response of standing still (F). By the end of the four epochs of Stage 1, the animal's knowledge, as expressed in terms of net values (Equation 8) can be summarized by the statement: Locomoting to $C$ located at $B$ yields $G$.

When shifted to training epochs where $C$ and $G$ are located at place $A$, the animal's knowledge becomes more difficult to summarize with a simple statement; it is more adequately described by considering Table 2. The net values (V́s) in Table 2 can be interpreted as the degree to which each event is predictively linked in an attentional-associative network with the goal G. The case we have been describing is that of a normal animal having experienced epochs as shown in Table 1. Table 2 shows that the net value of $C$ is greater than that of other events, $\mathrm{E}$ is next, followed by $\mathrm{X}, \mathrm{B}, \mathrm{A}$, and so forth. Notice also that $C$ has gained associatibility $\left(\alpha_{C}\right)$, while the other $\alpha$ s have either decreased or remained unchained.

If, at this point, $\mathrm{C}$ were moved to yet another location, one at which $\mathrm{C}$ was not obscure, that is, one that permitted the animal to know of C's presence and location on first entering the situation, the animal would locomote in the direction of $C$ "in anticipation of the goal." But what would the animal do if $\mathrm{C}$ were no longer present or was otherwise obscure? The animal would surely locomote, but to where? Since place B has a higher net value (.36) than place $A\left(\dot{V}_{A}=.29\right)$, the rat should go to place $B$, the place where the goal was first encountered. The reason that $B$ would be preferred to $A$ is that the predictive associative value of $C$ acquired in Stage 1 tended to block the development of the predictive associative value of $A$. This blocking is a consequence of the progressive loss of $\alpha_{A}$ over the epochs of Stage 2 of training by way of Equation 7 .

Elsewhere, we have argued (Moore \& Stickney, 1980) that disruption of normal brain functioning associated with lesions of the hippocampal function (e.g., hippocampal ablation) produces behavioral deficits because an animal so afflicted is less able than a normal animal to "tune out irrelevant events" (see, also, Cormier, 1981; Moore, 1979; Solomon, 1979, 1980; Solomon \& Moore, 1975). Moore and Stickney (1980) have linked this portrayal of dysfunction to the failure of Equation 7 to enter into computations of attentional-associative networks in the normal manner. Instead of decreasing $\alpha$ s where called for by Equation 7, as remain unchanged. This device has been successful in describing some of the pecu- liarities of animals with hippocampal damage in Pavlovian conditioning experiments (Moore \& Stickney, 1980). Accordingly, we simulated the development of the attention-associative network in our goal-tracking scenarios in the same way-all computations with Equation 7 produced zero; that is, there were no decreases in any of the $\alpha \mathrm{s}$. Increases of $\alpha \mathrm{s}$ by Equation 6 occurred normally.

Table 2 shows the knowledge possessed by a hippocampal animal after going through precisely the same epochs as did our previously described normal animal. Row by row comparisons of Vs show that both animals "believe that" locomoting to $C$ yields the goal. They differ, however, in the test case where $C$ is not present. Based on a comparison of $\dot{V}_{A}$ with $\dot{\mathrm{V}}_{\mathrm{B}}(.47 \mathrm{vs.} .35)$, the hippocampal animal will tend to go to place $A$ rather than place $B$. The simulation suggests that normal and hippocampal animals are equally able to follow a cue to a goal; they are equally capable of learning to make the appropriate responses to get there. The differences between the two animals is in their preference of place. This is precisely what one would expect from O'Keefe and Nadel's (1978) cognitive map theory of the hippocampus. To wit, their theory begins with the assumptions that hippocampal animals (1) can be guided by cues, as can normal animals, but (2) they possess altered "spatial strategies."

We have yet to illustrate how the behavior of the hippocampal animal can be disrupted by virtue of its greater potential than the normal animal's for going to place A instead of place B. A disruption or behavioral pathology would develop if the hippocampal animal were in conflict about where to go, that is, after the two stages of training (and without the guidance of $C$ ), $\dot{\mathrm{V}}_{\mathrm{A}}=\dot{\mathrm{V}}_{\mathrm{B}}$. We were able to simulate this state of affairs (Scenario-Sequence 2 of Tables 1-2) by a slight alteration of the scenario sequence. By disallowing "extinction" of place B in Stage $2, \dot{V}_{B}$ remains high enough to compete with $\dot{\mathrm{V}}_{\mathrm{A}}\left(\dot{\mathrm{V}}_{\mathrm{A}}=\dot{\mathrm{V}}_{\mathrm{B}}=.47\right)$. One way to prevent "extinction" of place B would be to force the animal away from this place during Stage 2.

Finally, it is instructive to consider a scenario sequence in which the normal and hippocampal animal are essentially alike in their preference for place $\mathbf{A}$ over place B. We simulated this outcome (ScenarioSequence 3 of Tables 1-2) by permitting remote associations of place A with the goal in Stage 1. Specifically, we assumed that the second bin of each Stage 1 epoch witnessed the animal locomoting in the vicinity of place $A$ en route to place $B$, where $C$ and $G$ werc located. With no opportunities for "extinction" of place $A$ prior to test, $\dot{\mathrm{V}}_{A}$ remains greater than $\dot{\mathrm{V}}_{\mathbf{B}}$ for both the normal and hippocampal animals. However, because of the comparatively high $\alpha_{A}$ value of .50 for the hippocampal animal compared with an $\alpha_{\mathrm{A}}$ of .18 for the normal animal, any "extinction" 
of place A would be more rapid for the hippocampal animal than for the normal animal, and as a consequence the hippocampal animal would soon find itself in conflict regarding place.

\section{Conclusion}

Deterministic models of associative learning provide a rigorous framework in which to view normal and pathological behavior. We have portrayed our model as a tool for analyzing particular instances of goal tracking as such behavior might evolve in any of a number of spatial learning tasks. We do not suggest that ours is the only model that lends itself to such an approach.

Simulations of our model illustrate the futility of attempting to characterize the effects of hippocampal damage, and perhaps other neuropsychological pathologies, using theories that are not formulated in terms of events encountered in real time. Simplistic as our model undoubtedly is, it nevertheless dramatizes the perils of attempting to predict the nature of differences in knowledge and behavior between normal and pathological cases in the absence of a complete record of their respective histories.

\section{REFERENCE NOTES}

1. Moore, J. W., \& Stickney, K. J. Conditioned inhibition in attentional-associative networks. In I. Gormezano, W. F. Prokasy, \& R. F. Thompson (Eds.), Classical conditioning III. In preparation.

2. Wagner, A. R. SOP: A model of automatic memory processing in animal behavior. Paper presented at the Binghamton Symposium on Memory Mechanisms in Animal Behavior, SUNYBinghamton, June 10-12, 1980.

\section{REFERENCES}

Black, A. H., Nadel, L., \& O'Keefe, J. Hippocampal function in avoidance learning and punishment. Psychological Bulletin, 1977, 84, 1107-1129.

Bush, R. R., \& Mosteller, F. Stochastic models for learning. New York: Wiley, 1955.

Cormier, S. M. A match-mismatch theory of limbic system function. Physiological Psychology, 1981, 9, 3-36.
Estes, W. K. The statistical approach to learning theory. In S. Koch (Ed.), Psychology: A study of a science (Vol. 2). New York: McGraw-Hill, 1959.

FreY, P. W., \& SEARS, R. J. Model of conditioning incorporating the Rescorla-Wagner associative axiom, a dynamic attention process, and a catastrophe rule. Psychological Review, 1978, 85, 321-340.

Mackintosh, N. J. A theory of attention: Variations in the associability of stimuli with reinforcement. Psychological Review, 1975, 82, 276-298.

Moore, J. W. Information processing in space-time by the hippocampus. Physiological Psychology, 1979, 7, 224-232.

Moore, J. W., \& Solomon, P. R. (Guest Editors) The role of the hippocampus in learning and memory. Physiological Psychology, 1980, 8, 145-296.

Moore, J. W., \& Stickney, K. J. Formation of attentionalassociative networks in real time: Role of the hippocampus and implications for conditioning. Physiological Psychology, 1980, 8, 207-217.

O'KeEFE, J., \& NADEL, L. The hippocampus as a cognitive map. Oxford: Oxford University Press, 1978.

Pearce, J. M., \& Hall, G. A model for Pavlovian learning: Variations in the effectiveness of conditioned but not of unconditioned stimuli. Psychological Review, 1980, 87, 532-552.

Resconla, R. A. Conditioned inhibition and extinction. In A. Dickinson \& R. A. Boakes (Eds.), Mechanisms of learning and motivation: A memorial volume to Jerzy Konorski. Hillsdale, N. J: Erlbaum, 1979.

Rescorla, R. A., \& Wagner, A. R. A theory of Pavlovian conditioning: Variations in the effectiveness of reinforcement and nonreinforcement. In A. H. Black \& W. F. Prokasy (Eds.), Classical conditioning II. New York: Appleton-Century-Crofts, 1972.

Solomon, P. R. Temporal versus spatial information processing theories of hippocampal function. Psychological Bulletin, 1979, 86, 1272-1279.

Solomon, P. R. A time and place for everything? Temporal processing views of hippocampal function with special reference to attention. Physiological Psychology, 1980, 8, 254-261.

Solomon, P. R., \& Moore, J. W. Latent inhibition and stimulus generalization of the classically conditioned nictitating mem-brane response in rabbits (Oryctolagus cuniculus) following dorsal hippocampal ablation. Journal of Comparative and Physiological Psychology, 1975, 89, 1192-1203.

Sutton, R. S., \& BARTo, A. G. Toward a modern theory of adaptive networks: Expectation and prediction. Psychological Review, 1981, 88, 135-170.

(Manuscript received July 6, 1981; revision accepted for publication January $11,1982$. 